\title{
Intermittierende Selbstbeatmung als Dauer- behandlung der chronisch respiratorischen Insuffizienz bei COPD - nichts ist wirklich bewiesen
}

\section{T. Köhnlein \\ T. Welte}

\author{
Non-Invasive Ventilation as Long-Term Treatment for Respiratory Insufficiency in \\ Severe COPD. Nothing is Really Proven
}

Die chronisch obstruktive Lungenerkrankung (COPD) ist eine langsam progrediente Erkrankung, an deren Ende eine chronisch respiratorische Globalinsuffizienz steht. Wenn dieses Stadium der Erkrankung erreicht wird, ist die weitere Prognose der Patienten schlecht. Anón [2] berichtete eine 1-Jahresmortalität von $75 \%$ und eine 5 -Jahressterblichkeit von $85 \%$, wenn Patienten mit schwerer COPD invasiv beatmungspflichtig werden. Dies deckt sich mit älteren Untersuchungen aus den siebziger Jahren [18].

Die etablierte medikamentöse Therapie mit Bronchodilatatoren (Anticholinergika, $\beta_{2}$-Agonisten, Theophyllin) hat nur symptomlindernden Charakter, ohne dass die Langzeitprognose der COPD wesentlich beeinflusst wird [3]. Auch der Versuch, die durch Granulozyten und aktivierte Alveolarmakrophagen getragene Entzündung durch Kortikosteroide zu günstig zu beeinflussen, muss als gescheitert angesehen werden (Übersicht bei [7]). Somit ist die Langzeitsauerstofftherapie bislang die einzige Maßnahme, die einen Überlebensvorteil für COPD-Patienten belegen konnte. Dies gilt allerdings nur dann, wenn sie mindestens 15 , besser mehr als 18 Stunden pro Tag angewendet wird $[13,14]$.

Je ausgeprägter jedoch die Hyperkapnie im Verlauf der Erkrankung wird, umso schwieriger wird eine ausreichend dosierte Sauerstofftherapie, da mit dem $\mathrm{PaO}_{2}$ gleichzeitig der $\mathrm{PaCO}_{2}$ ansteigt. Dies hat - neben negativen Effekten auf den Atemantrieb - vor allem Konsequenzen auf das kardiovaskuläre System. Kohlendioxid führt über eine Aktivierung von Chemorezeptoren zu einem Anstieg des peripheren und des pulmonalarteriellen Widerstands mit nachfolgender arterieller und pulmonaler Hypertonie, gleichzeitig steigt der Hirndruck an und es kommt zur Verschlechterung der zerebralen Perfusion [1]. Hyperkapnie

scheint die Prognose der Patienten direkt negativ zu beeinflussen [9].

Die Hyperkapnie des COPD-Patienten ist primär nicht die Folge der krankheitsbedingten Gasaustauschstörung, sondern aufgrund der Überblähung der Lunge kommt es zu einer Abflachung des Zwerchfells und zu einer Horizontalstellung der Rippen. Dadurch verschlechtert sich der Wirkungsgrad der Atemmuskulatur, der Energiebedarf der Muskulatur pro Atemzug erhöht sich, eine chronische Erschöpfung der Atemmuskelpumpe entwickelt sich. Hyperkapnie ist somit überwiegend die Folge einer alveolären Hypoventilation [16].

Neuromuskuläre Erkrankungen (Progressive Muskeldystrophien wie Morbus Duchenne; Schädigungen des Nervensystems wie bei Amyotropher Lateralsklerose, Guillain-Barré-Syndrom, u.a) und Wirbelsäulenfehlbildungen (BWS-Kyphoskoliose) gehen in ähnlicher Weise mit einer Erschöpfung der Atemmuskelpumpe und einer daraus resultierenden chronischen Hyperkapnie einher. Diese Erkrankungen werden seit etwa 10 Jahren erfolgreich mittels intermittierender Selbstbeatmung (ISB) über Gesichtsoder Nasenmasken behandelt. Die Entwicklung eines Cor pulmonale wird dadurch verhindert, ein bereits erhöhter pulmonaler Druck kann auf Dauer gesenkt werden, die Prognose der meist jungen Patienten bessert sich [17].

Von daher lag es nahe, das Prinzip der ISB auch auf die COPD mit respiratorischer Globalinsuffizienz zu übertragen. Dies wurde ab Mitte der 90er Jahre durch verschiedene Studien unterstützt, die bei akuter Exazerbation einer obstruktiven Atemwegserkrankung einen Nutzen von nicht-invasiver Beatmung unter Beweis stellen konnten. Mittels nicht-invasiver Beatmung gelang es

Institutsangaben

Bereich Pneumologie und Internistische Intensivmedizin, Otto-von-Guericke-

Universität Magdeburg, Magdeburg

Korrespondenzadresse

Priv.-Doz. Dr. med. T. Welte · Leiter des Bereiches Pneumologie und Internistische Intensivmedizin .

Otto-von-Guericke-Universität Magdeburg • Leipziger Str. 44 · 39120 Magdeburg

E-mail: tobias.welte@medizin.uni-magdeburg.de

Bibliografie

Pneumologie 2002; 56: 770-772 @ Georg Thieme Verlag Stuttgart · New York · ISSN 0934-8387 
meist rasch, Hypoxie und Hyperkapnie zu regulieren und damit eine Intubation des Patienten zu vermeiden [4]. Inzwischen wird ISB zunehmend bei COPD-Patienten eingesetzt; der Einsatz der Methode ist längst von spezialisierten Beatmungszentren auf Versorgungskrankenhäuser übergegangen, und auch die Kosten der Beatmung wurden ein nennenswerter Faktor in der Versorgung von COPD-Patienten [8].

Kann man aber aus den guten Langzeitergebnissen bei neuromuskulären Erkrankungen den gleichen Vorteil für Patienten mit COPD erwarten? Bedeutet therapeutischer Erfolg in der Akutphase gleichzeitig Prognoseverbesserung im Langzeitverlauf der chronisch respiratorischen Insuffizienz der COPD? Die Datenlage, die zur Beantwortung dieser Fragen herangezogen werden könnte, ist überraschend dürftig. Einer die ISB bei COPD unterstützenden Studie stehen 4 negative gegenüber. Alle Untersuchungen haben zudem erhebliche methodische Schwächen.

Meecham-Jones [12] untersuchte 18 Patienten mit schwerer COPD $\left(\mathrm{FEV}_{1} 0.86 \mathrm{l}\right.$, FVC $\left.2.03 \mathrm{l}\right)$ und respiratorischer Globalinsuffizienz $\left(\mathrm{PaO}_{2} 45 \mathrm{~mm} \mathrm{Hg}, \mathrm{PaCO}_{2} 56 \mathrm{~mm} \mathrm{Hg}\right)$ über 3 Monate crossover, zuerst Sauerstofftherapie alleine, dann nicht-invasive Beatmung (pressure support ventilation [PSV], inspiratorischer Druck im Mittel $20 \mathrm{~cm} \mathrm{H}_{2} \mathrm{O}$, exspiratorischer $4 \mathrm{~cm} \mathrm{H}_{2} \mathrm{O}$ ) plus Sauerstofftherapie. Er zeigt sowohl deutliche Veränderungen der Blutgase $\left(\mathrm{PaO}_{2}\right.$-Anstieg auf $50 \mathrm{~mm} \mathrm{Hg}, \mathrm{PaCO}_{2}$-Abfall auf $50 \mathrm{~mm} \mathrm{Hg}$ gegenüber dem Ausgangswert nach 12 Stunden Beatmungspause), als auch Verbesserungen der Schlaf- und der Lebensqualität. Problem der Studie war jedoch, dass 10 Patienten vor Studienbeginn Schlafstörungen aufwiesen, die durch die Therapie beseitigt wurden. Es konnte letztlich nicht ausgeschlossen werden, dass dies die Ergebnisse der Untersuchung wesentlich beeinflusst hat.

Strumpf [19] ventilierte in einer offenen, nicht kontrollierten Untersuchung 23 Patienten mit PSV und fand für keinen Parameter eine wesentliche Verbesserung. Allerdings beendeten nur $7 \mathrm{~Pa}-$ tienten die dreimonatige Studienphase, alle anderen tolerierten die Maske nicht. Zudem waren die Patienten mit einem $\mathrm{PaCO}_{2}$ von im Schnitt $46 \mathrm{~mm} \mathrm{Hg}$ mehrheitlich nicht hyperkapnisch.

Lin [10] zeigte ebenfalls keinen Vorteil für 10 Patienten, die in einer nicht kontrollierten Studie untersucht wurden, der Beobachtungszeitraum betrug jedoch nur 1 Monat.

Eine von der Studiengestaltung deutlich bessere Untersuchung wurde von Casanova [5] vor kurzem publiziert. Patienten mit stabiler, schwerer COPD ( $\mathrm{FEV}_{1} 0,82 \mathrm{l}$, FVC 2,12 1, $\mathrm{PaO}_{2} 55,7 \mathrm{~mm} \mathrm{Hg}$, $\mathrm{PaCO}_{2} 50,7 \mathrm{~mm} \mathrm{Hg}$ ) wurden randomisiert und entweder mit Sauerstofftherapie $(n=25)$ oder mit Sauerstofftherapie plus nichtinvasiver Beatmung ( $\mathrm{n}=26$, PSV, mittlere Drucke $12 / 4 \mathrm{~cm} \mathrm{H}_{2} \mathrm{O}$ ) versorgt. Evaluationen erfolgten nach 3 und 12 Monaten. Weder hinsichtlich Mortalität (22\% in beiden Gruppen), noch in der Exazerbationsrate, der Zahl der Krankenhausaufenthalte und der Notwendigkeit einer Intubation mit herkömmlicher Beatmung gab es Unterschiede zwischen den Gruppen.

Vor wenigen Wochen wurden die Ergebnisse einer multizentrisch angelegten, italienisch/französischen Studie über ISB bei Patienten mit stabiler COPD publiziert [6]. Diese ist die erste Stu- die, bei der eine größere Zahl von Patienten (122 eingeschlossen, 90 randomisiert) über einen längeren Zeitraum (mindestens zwei Jahre) prospektiv untersucht wurde. Eingeschlossen wurden Patienten, die seit mindestens sechs Monaten unter Langzeitsauerstoff-Therapie (LTOT) standen und in Ruhe bei Raumluftatmung einen $\mathrm{PaCO}_{2}>50 \mathrm{~mm} \mathrm{Hg}$ und einen $\mathrm{PaO}_{2}<60 \mathrm{~mm} \mathrm{Hg}$ aufwiesen. Sie wurden in zwei Gruppen, entweder LTOT alleine $(n=47)$ oder LTOT plus ISB $(n=43)$, randomisiert. ISB wurde immer im „pressure support ventilation“ (PSV)-Modus durchgeführt. Die Compliance zu LTOT war sehr gut, und ISB wurde mit durchschnittlich $9 \mathrm{~h} / \mathrm{Tag}$ ebenfalls gut von den Patienten akzeptiert. Die ISB-Gruppe wies nach einem Jahr kleine, statistisch zwar signifikante, klinisch jedoch unbedeutende Verbesserungen hinsichtlich Dyspnoeempfindung und gesundheitsbezogener Lebensqualität auf, nicht jedoch in der Mortalität oder in der Zahl der stationären Behandlungstage. Zwar zeigte sich ein Trend zu weniger Krankenhaustagen der ISB-Patienten und zu mehr Krankenhaustagen bei den LTOT-Patienten, beides erreichte jedoch keine statistische Signifikanz. Die Zahl der Behandlungstage auf der Intensivstation war in beiden Gruppen reduziert; sie war in der ISB-Gruppe noch tendenziell geringer als in der LTOT-Gruppe.

Kritisch anzumerken ist das sehr behutsame Vorgehen in der Beatmungseinstellung. Bereits eine Reduktion des Ausgangs- $\mathrm{PaCO}_{2}$ um $5 \%$ unter Beatmung im Wachzustand wurde als Beatmungserfolg angesehen. Die verwendeten Beatmungsdrucke von inspiratorisch $14 \pm 3 \mathrm{~cm} \mathrm{H}_{2} \mathrm{O}$ und exspiratorisch $2 \pm 1 \mathrm{~cm} \mathrm{H}_{2} \mathrm{O}$ sind für Patienten mit schwerer COPD wahrscheinlich zu niedrig. Ausschlaggebend für diese Einstellungen war der Komfort für den Patienten.

Das Problem aller Studien mit negativem Ausgang für ISB ist, dass die Patienten im Vergleich zu Meecham-Jones weniger hypoxisch und deutlich weniger hyperkapnisch waren. Die von Vitacca erst kürzlich vorgeschlagenen Kriterien für nicht-invasive Beatmung von COPD-Patienten [20] wurden von den meisten Patienten dieser Studien nicht erfüllt. Zudem wurden deutlich niedrigere Beatmungsdrücke (inspiratorisch $12 \mathrm{~cm} \mathrm{H}_{2} \mathrm{O}$ bei Casanova, $14 \mathrm{~cm} \mathrm{H}_{2} \mathrm{O}$ bei Clini, gegenüber $20 \mathrm{~cm} \mathrm{H}_{2} \mathrm{O}$ bei MeechamJones) gewählt. Geht man davon aus, dass etwa $50 \%$ des an der Maske applizierten Drucks aufgrund des erhöhten Atemwegswiderstands und des hohen Totraums verloren geht [15], erzielt man folglich kaum intratracheale Drucke, die geeignet erscheinen, eine ausreichende Ventilation herbeizuführen. Da sich jedoch der Erfolg der Beatmung bei COPD in erster Linie anhand der Senkung des $\mathrm{PaCO}_{2}$ abschätzen lässt [11], ist der Misserfolg dieser Untersuchungen vorgezeichnet.

Ein weiteres Problem der genannten Untersuchungen liegt darin, dass der Beobachtungszeitraum mit Ausnahme der Arbeiten von Casanova und Clini viel zu kurz war, um eine Veränderung der wesentlichen Endparameter Morbidität und Mortalität tatsächlich zu registrieren.

Zusammenfassend ist zu sagen, dass weder die Pro-, noch die Kontra-Untersuchungen überzeugen; letztlich fehlt es zum jetzigen Zeitpunkt an wissenschaftlichen Daten zur endgültigen Beurteilung von ISB als Dauerbehandlung bei COPD-Patienten. Insbesondere ist unklar, welche Patienten überhaupt für eine solche 
Therapieoption infrage kommen. Offen sind unter anderem folgende Fragen: $\mathrm{Ab}$ welchem $\mathrm{PaCO}_{2}$ soll die Beatmung beginnen? Dürfen nur hyperkapnische Patienten beatmet werden oder stellt die pulmonale Hypertonie, die ja die Prognose der Erkrankung entscheidend bestimmt [9], auch eine Indikation dar? Ist Letzteres gar eine Kontraindikation, weil durch Beatmung die rechtsventrikuläre Nachlast erhöht und der Ventrikel möglicherweise weiter belastet wird? Was ist das Beatmungsziel (Normalisierung des $\mathrm{PaCO}_{2}$ ? Senkung des pulmonalarteriellen Drucks? Verbesserung der Funktion der Atemmuskelpumpe? Verbesserung der Oxygenierung im Schlaf?) und mit welcher Beatmungseinstellung ist das zu erreichen? Welches sind die Zielparameter, die letztlich über den Erfolg der Methode entscheiden (Verbesserung der Mortalität? Erhöhung der Lebensqualität, Steigerung der Belastbarkeit?)? Nur eine große, multizentrische Studie wäre in der Lage, zumindest Teilantworten zu geben. Solche Untersuchungen sind teuer. Anders als die Pharmafirmen bei Medikamentenprüfungen, verfügt die Medizingeräteindustrie nicht über die Mittel, größere Studien zu finanzieren. In Anbetracht mangelnder Optionen zur Verbesserung der Prognose respiratorisch insuffizienter Patienten und der ökonomischen Brisanz, die mit dem breiten Einsatz nicht-invasiver Beatmungsverfahren verbunden ist, wäre es sicher sinnvoll, wenn eine solche Untersuchung entweder von den Kostenträgern selbst oder durch öffentliche Mittel unterstützt würde.

Zum jetzigen Zeitpunkt meinen wir, dass ein genereller Einsatz intermittierender Selbstbeatmung bei Patienten mit COPD nicht zu befürworten ist. Da „evidence-based medicine“ zu dieser Frage nicht zur Verfügung steht, sollte von einer Expertenkommission an erfahrenen Zentren festgelegt werden, bei welchen Patienten in Einzelfällen ein Therapieversuch unternommen werden sollte und wie der Therapieerfolg zu messen ist. Nur so kann vermieden werden, dass ein theoretisch überzeugender Therapieansatz durch falsche Anwendung generell in Misskredit gerät.

\section{Literatur}

${ }^{1}$ Aalkjaer C, Poston L. Effects of $\mathrm{pH}$ on vascular tension: which are the important mechanisms? J Vasc Res 1996; 33: 347 - 359

2 Anón JM, Garcia de Lorenzo A, Zarazaga A et al. Mechanical ventilation of patients on long-term oxygen therapy with acute exacerbations of chronic obstructive pulmonary disease: prognosis and cost-utility analysis. Intensive Care Med 1999; 25: $452-457$

${ }^{3}$ Anthonisen NR, Connett JE, Kiley JP et al. Effects of smoking intervention and the use of an inhaled anticholinergic bronchodilator on the rate of decline of $\mathrm{FEV}_{1}$. Lung Health Study JAMA 1994; 272: $1497-1505$
${ }^{4}$ Brochard L, Mancebo J, Wysocki M et al. Noninvasive ventilation for acute exacerbations of chronic obstructive pulmonary disease. $\mathrm{N}$ Engl J Med 1995; 333: 817-822

${ }^{5}$ Casanova C, Celli BR, Tost T et al. Long-term Controlled Trial of Nocturnal Nasal Positive Pressure Ventilation in Patients With Severe COPD. Chest 2000; 118: $1582-1590$

${ }^{6}$ Clini E, Sturani C, Rossi A et al. On behalf of the Rehabilitation and Chronic Care Study Group, Italian Association of Hospital Pulmonologists (AIPO). The Italian mulitcentre study on noninvasive ventilation in chronic obstructive pulmonary disease patients. Eur Respir J 2002; 20: $529-538$

${ }^{7}$ Gillissen A, Barczok M, Buhl R et al. Inhalierbare Kortikosteroide in der Langzeittherapie der COPD. Pneumologie 2000; 54: 306-308

${ }^{8}$ Keenan SP, Kernerman PD, Cook DJ et al. Effect of noninvasive positive pressure ventilation on mortality in patients admitted with acute respiratory failure: A meta-analysis. Crit Care Med 1997; 25: $1685-1692$

${ }^{9}$ Kessler R, Faller M, Fourgaut G et al. Predictive factors of hospitalization for acute exacerbation in a series of 64 patients with chronic obstructive pulmonary disease. Am J Respir Crit Care Med 1999; 159; $158-164$

${ }^{10}$ Lin CC. Comparison between nocturnal nasal positive pressure ventilation combined with oxygen therapy and oxygen monotherapy in patients with severe COPD. Am J Respir Crit Care Med 1996; 154: $353-358$

${ }^{11}$ Meduri GU, Turner RE, Abou-Shala N et al. Noninvasive positive pressure ventilation via face mask. First line intervention in patients with acute hypercapnic and hypoxemic respiratory failure. Chest 1996; 109: $179-193$

12 Meecham Jones DJ, Paul EA, Jones PW et al. Nasal pressure support ventilation plus oxygen compared with oxygen therapy alone in hypercapnic COPD. Am J Resp Crit Care Med 1995; 152: 538 - 544

13 Nocturnal oxygen therapy trial group. Continous or nocturnal oxygen therapy in hypoxemic chronic obstructive pulmonary disease. Ann Intern Med 1980; 93: $191-198$

${ }^{14}$ Report of the Medical Research Council Working Party. Long-term domiciliary oxygen in chronic cor pulmonale complicating chronic bronchitis and emphysema. Lancet 1981; 1: 681 -685

${ }^{15}$ Robinson TD, Freiberg DB, Regnis JA et al. The role of hypoventilation and ventilation-perfusion redistribution in oxygen-induced hypercapnia during acute exacerbations of chronic obstructive pulmonary disease. Am J Respir Crit Care Med 2000; 161: 1524-1529

${ }^{16}$ Schönhofer B, Köhler D. Ventilatorische Insuffizienz und hyperkapnische Kompensation infolge chronisch belasteter Atempumpe. Physiologie, Pathophysiologie und Therapie. Dtsch Med Wschr 1994; 119: $1209-1214$

${ }^{17}$ Simonds AK, Elliott MW. Outcome of domiciliary nasal intermittent positive pressure ventilation in restrictive and obstructive disorders. Thorax 1995; 50: 604-609

${ }^{18}$ Sluiter HJ, Blokzijl EF, van Dijl W et al. Conservative and respirator treatment of acute respiratory insufficiency in patients with chronic obstructive lung disease. A reappraisal. Am Rev Respir Dis 1972; 105: $932-943$

${ }^{19}$ Strumpf DA, Millman RP, Carlisle CC et al. Nocturnal positive-pressure ventilation via nasal mask in patients with severe chronic obstructive pulmonary disease. Am Rev Respir Dis 1991; 144: 1234-1239

20 Vitacca M, Nava S, Gonfalonieri M et al. The appropriate setting of noninvasive pressure support ventilation in stable COPD patients. Chest 2000; 118: 1286-1293 